\title{
Optimal Dynamic Portfolio Selection with Earnings-at-Risk
}

\author{
Zhong-Fei Li ${ }^{1}$, Hailiang Yang${ }^{2}$, And XiaO-Tie Deng ${ }^{3}$
}

\begin{abstract}
In this paper we investigate a continuous-time portfolio selection problem. Instead of using the classical variance as usual, we use Earnings-at-Risk (EaR) of terminal wealth as a measure of risk. In the settings of Black-Scholes type financial markets and constantrebalanced portfolio (CRP) investment strategies, we obtain closedform expressions for the best CRP investment strategy and the efficient frontier of the mean-EaR problem, and compare our mean-EaR analysis to the classical mean-variance analysis, to the mean-CaR analysis in Ref. 13, and to the expected utility analysis. We also examine some economic implications arising from using the mean-EaR model.
\end{abstract}

Keywords. Dynamic portfolio optimization, Earnings-at-Risk, constant-rebalanced portfolios, Black-Scholes financial market.

\footnotetext{
${ }^{1}$ To whom correspondence should be addressed. Professor, Department of Finance, Lingnan (University) College, Sun Yat-Sen University, Guangzhou 510275, People's Republic of China. The research of this author was supported in part by a grant of the National Excellent Ph.D. Thesis Project of China (No. 200267), a grant of the National Natural Science Foundation of China (No. 10171115), a "Tenth Five-Year Plan" project of Ministry of Education of China (No. 01JA630009), and a grant of the Natural Science Foundation of Guangdong Province (No. 011193)

${ }^{2}$ Associate Professor, Department of Statistics and Actuarial Science, The University of Hong Kong, Pokfulam Road, Hong Kong. The research of this author was supported in part by a research grant of Hong Kong Research Grant Council (HKU 7139/01H) and a research grant of The University of Hong Kong.

${ }^{3}$ Professor, Department of Computer Science, City University of Hong Kong, Kowloon, Hong Kong. The research of this author was supported in part by a research grant of Hong Kong Research Grant Council and a research grant of City University of Hong Kong.
} 


\section{Introduction}

The pioneering work of Markowitz in Ref. 1 introduced the mean-variance framework for portfolio selection and risk management which are important problems in investment finance. The mean-variance approach has become the foundation of modern finance theory and inspired literally a substantial number of extensions and applications. From a theoretical point of view, two challenges can be identified. The first is the extension of the classical single-period mean-variance analysis to a multi-period or continuous-time mean-variance analysis. A large part of literature focuses on maximizing some timeadditive utility of terminal wealth and/or consumption; see, e.g., Refs. 2-5. However, enormous difficulties in solving dynamic mean-variance problems was reported; see, e.g., Ref. 6. Consequently, Markowitz's mean-variance formulation has not been fully exploited in dynamic cases for a long time. Only in recent years, the dynamic meanvariance problems have been solved analytically by $\mathrm{Li}$ and $\mathrm{Ng}$ in Ref. 7 and Zhou and $\mathrm{Li}$ in Ref. 8, respectively, in a discrete-time and a continuous-time frameworks.

The second challenge lies on appropriate measure of risk. While there is no ambiguity on the definition of return, the measure of risk is rather subjective. Consequently, many variants of risk measures have been proposed. These include absolute deviation, semivariance, shortfall probability, safety-first, etc. Many of these measures are typically based on the notion of downside risk concepts such as the lower partial moments. More recently, some new risk measures such as the value at risk (VaR) (see Ref. 9), the coherence risk (see Ref. 10) and the limited expected loss (see Ref. 11) have been advocated. Despite its several drawback, VaR remains the most prominent risk measure and its importance continues to grow since regulators accept it as a benchmark for controlling market risk.

Besides its uses as a potential risk measure, VaR has also been applied in the context of portfolio selection. For instance, in Refs. 12 and 13, the authors define a VaR-based related concept known as Capital at Risk $(\mathrm{CaR})$ and demonstrate how to incorporate such measure in the portfolio optimization problem. Using a constant-rebalanced portfolio (CRP) investment strategy, they formulated a mean-CaR portfolio optimization problem and derived analytically the optimal solution and the efficient portfolio frontier for the problem. A CRP strategy is an investment strategy which keeps the same distribution of wealth among a set of securities from time to time (or from period to 
period). That is, the proportion of total wealth invested in each of the underlying securities is the same at any time point (or period); see, for example, Refs. 12-15. It should be emphasized that such strategy does not imply that there is no trading. As the stock prices evolve randomly one has to trade at every instant to ensure the fraction of wealth invested in each security constant. Thus, following a CRP investment strategy still means one must trade dynamically.

In order to demonstrate the power of constant-rebalanced portfolio investment strategies, we cite the example in Ref. 15. Assume that only two securities are available. The first one is riskless, whose price never changes. The second is highly volatile, whose price doubles on even days and halves on odd days. Thus the price processes can be described by the sequence $\{1,1,1, \ldots$,$\} for the first stock and by \left\{\frac{1}{2}, 2, \frac{1}{2}, 2, \ldots,\right\}$ for the second. Neither investing a single stock can increasing its wealth by more than a factor of 2. However, a constant-rebalanced portfolio $\left(\frac{1}{2}, \frac{1}{2}\right)$ will increase its wealth exponentially. The investment strategy trades stocks so that it has an equal wealth in each stock at the beginning of each day and maintains this until the end of the day. On odd days the total wealth will decrease by a factor of $\frac{1}{2} \times 1+\frac{1}{2} \times \frac{1}{2}=\frac{3}{4}$ and on even days will increases by $\frac{1}{2} \times 1+\frac{1}{2} \times 2=\frac{3}{3}$. Thus, after two consecutive trading days the investor's wealth will grows by a factor of $\frac{3}{4} \times \frac{3}{2}=\frac{9}{8}$. It takes only twelve trading days to double the wealth, and over $2 n$ days the wealth increases by a factor of $\left(\frac{9}{8}\right)^{n}$.

In this paper, we investigate a dynamic portfolio selection problem in the framework of (i) the Black-Scholes type financial market, (ii) a CRP investment strategy, and (iii) a mean-EaR tradeoff. Section 2 describes the financial market which involves the BlackScholes settings and CRP investment strategies, and introduces a risk measure known as Earnings-at-Risk (EaR). Some properties of EaR are provided in Section 3. Section 4 establishes a mean-EaR portfolio optimization model and derives analytically its optimal solution and efficient frontier. A comparison with the classic mean-variance analysis, the mean-CaR analysis, and the expected utility analysis is given in Section 5. Section 6 concludes the paper.

\section{The Financial Market and EaR}

Consider a standard Black-Scholes type financial market in which $n+1$ assets (or securities) are traded continuously in the horizon $[0, T]$ and indexed by $i=0,1, \ldots, n$. One of the assets, say $i=0$, is the riskless bond whose price process $P_{0}(t)$ evolves 
according to the following (deterministic) ordinary differential equation

$$
d P_{0}(t)=P_{0}(t) r d t \quad \text { for } \quad t \in[0, T]
$$

where $r$ is the rate of interest and is assumed to be constant. The other $n$ assets are risky stocks whose price processes $P_{1}(t), \ldots, P_{n}(t)$ follow the following stochastic differential equations

$$
d P_{i}(t)=P_{i}(t)\left(b_{i} d t+\sum_{j=1}^{n} \sigma_{i j} d B_{j}(t)\right) \quad \text { for } \quad t \in[0, T], \quad i=1, \ldots, n,
$$

where $b=\left(b_{1}, \ldots, b_{n}\right)^{\prime}$ is the vector of stock-appreciation rate, $\sigma=\left(\sigma_{i j}\right)_{n \times n}$ is the matrix of stock-volatilities and $B(t)=\left(B_{1}(t), \ldots, B_{n}(t)\right)^{\prime}$ is a standard $n$-dimensional Brownian motion. Here $b$ and $\sigma$ are assumed to be constant in time. Moreover, for simplicity, we assume that $\sigma$ is invertible and that $b_{i} \geq r$.

Let $\pi_{i}(t)$ be the fraction of the wealth $W^{\pi}(t)$ invested in asset $i$ at time $t$. Let $\pi(t)=\left(\pi_{1}(t), \ldots, \pi_{n}(t)\right)^{\prime} \in \mathbb{R}^{n}$. Then $\pi_{0}(t)=1-\pi(t)^{\prime} \mathbf{1}$, where $\mathbf{1}=(1, \ldots, 1)^{\prime}$ is the vector whose components are all units. The portfolio process $\pi(t)$ is called a portfolio strategy. The number of shares at time $t$ invested in asset $i$ is

$$
\begin{aligned}
& N_{0}(t)=W^{\pi}(t)\left(1-\pi(t)^{\prime} \mathbf{1}\right) / P_{0}(t), \quad i=0, \\
& N_{i}(t)=W^{\pi}(t) \pi_{i}(t) / P_{i}(t), \quad i=1, \ldots, n .
\end{aligned}
$$

Hence,

$$
W^{\pi}(t)=\sum_{i=0}^{n} N_{i}(t) P_{i}(t)
$$

Throughout the paper, we assume that transaction costs and consumption are not considered and that portfolio strategy $\pi(t)$ is self-financing. Thus

$$
\begin{aligned}
d W^{\pi}(t) & =\sum_{i=0}^{n} N_{i}(t) d P_{i}(t) \\
& =\left\{r N_{0}(t) P_{0}(t)+\sum_{i=1}^{n} b_{i} N_{i}(t) P_{i}(t)\right\} d t+\sum_{i=1}^{n} N_{i}(t) P_{i}(t) \sum_{j=1}^{n} \sigma_{i j} d B_{j}(t) \\
& =W^{\pi}(t)\left\{\left(\left(1-\pi(t)^{\prime} \mathbf{1}\right) r+\pi(t)^{\prime} b\right) d t+\pi(t)^{\prime} \sigma d B(t)\right\}
\end{aligned}
$$

with $W^{\pi}(0)=w>0$ being the initial wealth of an investor.

As in Refs. 12-15 and many others, in what follows we restrict ourselves to constantrebalanced portfolio (CRP) strategies. ${ }^{4}$ As noted in the introduction, a CRP strategy

\footnotetext{
${ }^{4}$ In Refs. 2 and 3, Merton showed that this form of strategies are optimal to portfolio selection problems of maximizing expected utility with constant relative risk-aversion.
} 
will be rebalanced at each time instant so that a fixed fraction of the wealth is held in each of the underlying stocks. Therefore, a CRP strategy employs the same investment vector $\pi(t)=\pi=\left(\pi_{1}, \ldots, \pi_{n}\right)^{\prime}$ at each $t$ in the planning horizon $[0, T]$. Such an investment strategy still means that one must follow a dynamic trading strategy, since at each time instant $t$ the investment proportions are rebalanced back to the vector $\pi$. Even it might result in vast amounts of trading. The advantage of CRP strategies is two-fold: first we obtain, at least in a Black-Scholes setting, closed-form results; and, furthermore, the economic interpretation of the mathematical results is comparably easy.

Standard Itô integral and the fact that $E\left[e^{s B_{j}(t)}\right]=e^{t s^{2} / 2}$, where $E$ is the expectation operator, yields the following explicit formulae for the wealth process $W^{\pi}(t)$ for all $t \in[0, T]$ (see, e.g., Ref. 13).

$$
\begin{aligned}
W^{\pi}(t) & =w \exp \left(\left(\pi^{\prime}(b-r \mathbf{1})+r-\left\|\pi^{\prime} \sigma\right\|^{2} / 2\right) t+\pi^{\prime} \sigma B(t)\right), \\
E\left[W^{\pi}(t)\right] & =w \exp \left(\left(\pi^{\prime}(b-r \mathbf{1})+r\right) t\right), \\
\operatorname{Var}\left[W^{\pi}(t)\right] & =w^{2} \exp \left(2\left(\pi^{\prime}(b-r \mathbf{1})+r\right) t\right)\left(\exp \left(\left\|\pi^{\prime} \sigma\right\|^{2} t\right)-1\right),
\end{aligned}
$$

where $\|\cdot\|$ denotes the Euclidean norm in $\mathbb{R}^{n}$ and $\operatorname{Var}$ is the variance operator.

Associated with real number $\alpha \in(0,1)$, initial wealth $w$, time horizon $T$ and portfolio $\pi$, we denote by $\rho_{0}(\pi, w, T)$ the $\alpha$-quantile of the terminal wealth $W^{\pi}(T)$, that is, it is implicitly defined by

$$
P\left(W^{\pi}(T) \leq \rho_{0}(\pi, w, T)\right)=\alpha,
$$

where $P(\cdot)$ is the probability. Using the notation $\rho_{0}$, the expected shortfall or more precisely the conditional tail expectation of $W^{\pi}(T)$ is defined as

$$
\rho_{1}(\pi, w, T)=E\left[W^{\pi}(T) \mid W^{\pi}(T) \leq \rho_{0}(\pi, w, T)\right]
$$

Furthermore, the conditional tail semi-standard derivation of $W^{\pi}(T)$ is defined as

$$
\rho_{2}(\pi, w, T)=\sqrt{E\left[\left(W^{\pi}(T)\right)^{2} \mid W^{\pi}(T) \leq \rho_{0}(\pi, w, T)\right]}
$$

Using the risk measures $\rho_{k}(\pi, w, T), k=0,1,2$, we can define a class of Earningsat-Risk.

Definition 2.1 (Earnings-at-Risk). Earnings-at-Risk (EaR) of a CRP investment strategy $\pi$ with respect to $\rho_{k}(k=0,1,2)$ with initial wealth $w$ and time horizon $T$ is 
the difference between the mean terminal wealth $E\left[W^{\pi}(T)\right]$ and the risk measure $\rho_{k}$, i.e.,

$$
\operatorname{EaR}_{k}(\pi, w, T):=E\left[W^{\pi}(T)\right]-\rho_{k}(\pi, w, T) .
$$

Note that there are important distinctions between the proposed EaR and the Capital-at-Risk (CaR) defined in Refs. 12 and 13. CaR is defined as the difference between the terminal wealth of the pure bond (riskless) investment strategy and the risk measure $\rho_{k}(\pi)$. EaR measures risk relative to mean terminal wealth $E\left[W^{\pi}(T)\right]$ while CaR measures risk relative to pure bond investment strategy. The mean terminal wealth depends explicitly on the adopted investment strategy $\pi$ while the pure bond strategy is independent of $\pi$. EaR therefore provides a trade-off between investing in the portfolio with position $\pi$ and its expected shortfall as a result of adopting such investment strategy. When formulated as an optimization problem, both the mean return and its risk measure are considered jointly. Hence it is a more relevant measure over $\mathrm{CaR}$ which provides a trade-off between the risk-free investment and its associated risk measure.

Let $z_{\alpha}$ be the $\alpha$-quantile of the standard normal distribution and $\Phi$ the distribution function of a standard normal random variable.

Since $\pi^{\prime} \sigma B(T) /\left(\left\|\pi^{\prime} \sigma\right\| \sqrt{T}\right)$ is a standard normal random variable, by using (2.1) and (2.4)-(2.7) we can express explicitly the risk measures $\rho_{k}, k=0,1,2$ as (see Ref. 12)

$$
\begin{aligned}
& \rho_{0}(\pi, w, T)=w \exp \left(\left(\pi^{\prime}(b-r \mathbf{1})+r-\left\|\pi^{\prime} \sigma\right\|^{2} / 2\right) T+z_{\alpha}\left\|\pi^{\prime} \sigma\right\| \sqrt{T}\right), \\
& \rho_{1}(\pi, w, T)=w \exp \left(\left(\pi^{\prime}(b-r \mathbf{1})+r\right) T\right) \frac{\Phi\left(z_{\alpha}-\left\|\pi^{\prime} \sigma\right\| \sqrt{T}\right)}{\alpha}, \\
& \rho_{2}(\pi, w, T)=w \exp \left(\left(\pi^{\prime}(b-r \mathbf{1})+r+\left\|\pi^{\prime} \sigma\right\|^{2} / 2\right) T\right) \sqrt{\frac{\Phi\left(z_{\alpha}-2\left\|\pi^{\prime} \sigma\right\| \sqrt{T}\right)}{\alpha}} .
\end{aligned}
$$

Consequently, closed-form expressions of $E a R_{k}$ for $k=0,1,2$ are respectively given by

$$
\begin{aligned}
& \operatorname{EaR}_{0}(\pi)=w \exp \left(\left(\pi^{\prime}(b-r \mathbf{1})+r\right) T\right)\left[1-\exp \left(z_{\alpha}\left\|\pi^{\prime} \sigma\right\| \sqrt{T}-\left\|\pi^{\prime} \sigma\right\|^{2} T / 2\right)\right], \\
& \operatorname{EaR}_{1}(\pi)=w \exp \left(\left(\pi^{\prime}(b-r \mathbf{1})+r\right) T\right)\left[1-\frac{\Phi\left(z_{\alpha}-\left\|\pi^{\prime} \sigma\right\| \sqrt{T}\right)}{\alpha}\right], \\
& \operatorname{EaR}_{2}(\pi)=w \exp \left(\left(\pi^{\prime}(b-r \mathbf{1})+r\right) T\right)\left[1-\sqrt{\exp \left(\left\|\pi^{\prime} \sigma\right\|^{2} T\right) \frac{\Phi\left(z_{\alpha}-2\left\|\pi^{\prime} \sigma\right\| \sqrt{T}\right)}{\alpha}}\right] .
\end{aligned}
$$

Here and hereafter we simply use $\operatorname{EaR}_{k}(\pi)$ to stand for $\operatorname{EaR}_{k}(\pi, w, T)$ for $k=0,1,2$. 
To avoid some subcases in the results of this paper, we make the following assumption.

Assumption 2.1. The parameter $\alpha$ satisfies $\alpha<0.5$ and hence $z_{\alpha}<0$.

\section{Some Properties of EaR}

The three $E a R_{k}$ 's have the following relations.

Proposition 3.1. For any portfolio $\pi$, initial wealth $w$ and time horizon $T$,

(1) $\rho_{1}(\pi, w, T) \leq \rho_{2}(\pi, w, T) \leq \rho_{0}(\pi, w, T)$.

(2) $\operatorname{EaR}_{0}(\pi, w, T) \leq \operatorname{EaR}_{2}(\pi, w, T) \leq \operatorname{EaR}_{1}(\pi, w, T)$.

(3) For $k=0,1,2, \operatorname{EaR}_{k}(\pi, w, T) \begin{cases}=0 & \text { if } \pi=0, \\ >0 & \text { if } \pi \neq 0 .\end{cases}$

Proof. For (1) see Ref. 12. Assertion (2) follows from (1) and Definition 2.1. Assertion

(3) follows from (2), (2.11)-(2.13) and the assumption that matrix $\sigma$ is invertible.

Denote by $\varphi$ the density function of a standard normal random variable.

Lemma 3.2. Let $x>0$. Then

$$
\left(\frac{1}{x}-\frac{1}{x^{3}}\right) \varphi(x)<\Phi(-x)<\frac{\varphi(x)}{x} .
$$

Proof. See Ref. 16.

Define two functions $g_{1}$ and $g_{2}$ on $(0,+\infty)$ by

$$
g_{1}(\varepsilon):=\frac{1}{\alpha} \exp \left(\varepsilon^{2} T\right) \Phi\left(z_{\alpha}-2 \varepsilon \sqrt{T}\right)
$$

and

$$
g_{2}(\varepsilon):=\varepsilon \theta T+r T+\ln \left(1-\sqrt{\exp \left(\varepsilon^{2} T\right) \frac{\Phi\left(z_{\alpha}-2 \varepsilon \sqrt{T}\right)}{\alpha}}\right) .
$$

respectively, where $\theta=\left\|\sigma^{-1}(b-r \mathbf{1})\right\|$. The following properties of these two functions will be used in the sequel.

Lemma 3.3. The following statements are true:

(1) $g_{1}$ is strictly decreasing, $g_{1}(\varepsilon) \in(0,1)$ for all $\varepsilon \in(0,+\infty)$, and

$$
\lim _{\varepsilon \rightarrow 0^{+}} g_{1}(\varepsilon)=1, \quad \lim _{\varepsilon \rightarrow+\infty} g_{1}(\varepsilon)=0 .
$$

(2) $g_{2}$ is strictly increasing, $g_{1}(\varepsilon) \in(-\infty,+\infty)$ for all $\varepsilon \in(0,+\infty)$, and

$$
\lim _{\varepsilon \rightarrow 0^{+}} g_{2}(\varepsilon)=-\infty, \quad \lim _{\varepsilon \rightarrow+\infty} g_{2}(\varepsilon)=+\infty
$$


Proof. Let $\varepsilon \in(0,+\infty)$. Clearly $g_{1}(\varepsilon)>0$. Since $\operatorname{EaR}_{2}(\pi, w, T)>0$ for all $\pi \neq 0$ by Proposition 3.1 (3), it follows that $g_{1}(\varepsilon)<1$. Noting $1-\Phi(x)=\Phi(-x)$ and $\varphi(-x)=\varphi(x)$, setting $x=2 \varepsilon \sqrt{T}-z_{\alpha}$ in the second inequality in Lemma 3.2 yields $\varphi\left(z_{\alpha}-2 \varepsilon \sqrt{T}\right)>\Phi\left(z_{\alpha}-2 \varepsilon \sqrt{T}\right)\left(2 \varepsilon \sqrt{T}-z_{\alpha}\right)$. Thus we have

$$
\begin{aligned}
g_{1}^{\prime}(\varepsilon) & =\frac{1}{\alpha} \exp \left(\varepsilon^{2} T\right)(2 \varepsilon T) \Phi\left(z_{\alpha}-2 \varepsilon \sqrt{T}\right)+\frac{1}{\alpha} \exp \left(\varepsilon^{2} T\right) \varphi\left(z_{\alpha}-2 \varepsilon \sqrt{T}\right)(-2 \sqrt{T}) \\
& =\frac{2 \sqrt{T}}{\alpha} \exp \left(\varepsilon^{2} T\right)\left[\varepsilon \sqrt{T} \Phi\left(z_{\alpha}-2 \varepsilon \sqrt{T}\right)-\varphi\left(z_{\alpha}-2 \varepsilon \sqrt{T}\right)\right] \\
& <\frac{2 \sqrt{T}}{\alpha} \exp \left(\varepsilon^{2} T\right)\left[\varepsilon \sqrt{T} \Phi\left(z_{\alpha}-2 \varepsilon \sqrt{T}\right)-\Phi\left(z_{\alpha}-2 \varepsilon \sqrt{T}\right)\left(2 \varepsilon \sqrt{T}-z_{\alpha}\right)\right] \\
& =\frac{2 \sqrt{T}}{\alpha} \exp \left(\varepsilon^{2} T\right) \Phi\left(z_{\alpha}-2 \varepsilon \sqrt{T}\right)\left(-\varepsilon \sqrt{T}-\left|z_{\alpha}\right|\right) \\
& <0 .
\end{aligned}
$$

Hence $g_{1}(\varepsilon)$ is strictly decreasing on $(0,+\infty)$, and hence $g_{2}(\varepsilon)$ is strictly increasing on $(0,+\infty)$ because $g_{2}(\varepsilon)=\varepsilon \theta T+r T+\ln \left(1-\sqrt{g_{1}(\varepsilon)}\right)$. Obviously, $\lim _{\varepsilon \rightarrow 0^{+}} g_{1}(\varepsilon)=1$. By using $\varphi^{\prime}(x)=(-x) \varphi(x)$ and L'Hopital, we have

$$
\begin{aligned}
\lim _{\varepsilon \rightarrow+\infty} g_{1}(\varepsilon) & =\lim _{\varepsilon \rightarrow+\infty} \frac{1}{\alpha} \exp \left(\varepsilon^{2} T\right) \Phi\left(z_{\alpha}-2 \varepsilon \sqrt{T}\right) \\
& =\lim _{\varepsilon \rightarrow+\infty} \frac{\Phi\left(z_{\alpha}-2 \varepsilon \sqrt{T}\right)}{\alpha \exp \left(-\varepsilon^{2} T\right)} \\
& =\lim _{\varepsilon \rightarrow+\infty} \frac{\varphi\left(z_{\alpha}-2 \varepsilon \sqrt{T}\right)\left(2 \varepsilon \sqrt{T}-z_{\alpha}\right)(-2 \sqrt{T})}{-2 T \varepsilon \alpha \exp \left(-\varepsilon^{2} T\right)} \\
& =\lim _{\varepsilon \rightarrow+\infty} \frac{2 \varepsilon \sqrt{T}-z_{\alpha}}{\varepsilon \sqrt{T}} \frac{\exp \left(\varepsilon^{2} T\right)}{\sqrt{2 \pi} \exp \left(\frac{1}{2}\left(z_{\alpha}-2 \varepsilon \sqrt{T}\right)^{2}\right)} \\
& =\lim _{\varepsilon \rightarrow+\infty} \frac{2 \varepsilon \sqrt{T}-z_{\alpha}}{\varepsilon \sqrt{T}} \frac{1}{\sqrt{2 \pi} \exp \left(\frac{1}{2} z_{\alpha}^{2}-2 z_{\alpha} \varepsilon \sqrt{T}+\varepsilon^{2} T\right)}=0
\end{aligned}
$$

Hence,

$$
\lim _{\varepsilon \rightarrow+\infty} g_{2}(\varepsilon)=\lim _{\varepsilon \rightarrow+\infty} \varepsilon \theta T+r T+\ln \left(1-\sqrt{g_{1}(\varepsilon)}\right)=+\infty .
$$

Lastly, $\lim _{\varepsilon \rightarrow 0^{+}} g_{2}(\varepsilon)=-\infty$ is evident.

Now we give a extreme property of Earnings-at-Risk.

Proposition 3.4. For $k=0,1,2$,

(1) $\sup _{\pi \in \mathbb{R}^{n}} \operatorname{EaR}_{k}(\pi)= \begin{cases}w e^{r T} & \text { if } b=r \mathbf{1}, \\ +\infty & \text { otherwise. }\end{cases}$

(2) $\min _{\pi \in \mathbb{R}^{n}} \operatorname{EaR}_{k}(\pi)=0$ and the minimum is only attained for the pure bond strategy. 
Proof. We show only the case of $k=2$.

(1) If $b=r \mathbf{1}$, the conclusion is obvious. Now we assume that $b \neq r \mathbf{1}$. We rewrite the expression (2.13) of $E a R_{2}$ in the following form:

$$
\operatorname{EaR}_{2}(\pi, w, T)= \begin{cases}w e^{f(\pi)} & \text { if }\left\|\pi^{\prime} \sigma\right\|>0 \\ 0 & \text { if }\left\|\pi^{\prime} \sigma\right\|=0\end{cases}
$$

where

$$
f(\pi)=\left(\pi^{\prime}(b-r \mathbf{1})+r\right) T+\ln \left(1-\sqrt{\exp \left(\left\|\pi^{\prime} \sigma\right\|^{2} T\right) \frac{\Phi\left(z_{\alpha}-2\left\|\pi^{\prime} \sigma\right\| \sqrt{T}\right)}{\alpha}}\right) .
$$

Now consider the following optimization problem

$$
\max _{\pi} \quad f(\pi) \quad \text { subject to } \quad\left\|\pi^{\prime} \sigma\right\|=\varepsilon
$$

for any given $\varepsilon>0$. Over the (boundary of the) ellipsoid defined by the constraint in problem (3.1), the objective function equals

$$
f(\pi)=\left(\pi^{\prime}(b-r \mathbf{1})+r\right) T+\ln \left(1-\sqrt{\exp \left(\varepsilon^{2} T\right) \frac{\Phi\left(z_{\alpha}-2 \varepsilon \sqrt{T}\right)}{\alpha}}\right) .
$$

Hence, solving problem (3.1) is equivalent to solving the following problem

$$
\max _{\pi} \pi^{\prime}(b-r \mathbf{1}) \quad \text { subject to } \quad \pi^{\prime}\left(\sigma \sigma^{\prime}\right) \pi=\varepsilon^{2} .
$$

Using the Lagrangian method, this problem has the unique optimal solution

$$
\pi_{\varepsilon}^{*}=\varepsilon \frac{\left(\sigma \sigma^{\prime}\right)^{-1}(b-r \mathbf{1})}{\left\|\sigma^{-1}(b-r \mathbf{1})\right\|}
$$

with

$$
f\left(\pi_{\varepsilon}^{*}\right)=\varepsilon \theta T+r T+\ln \left(1-\sqrt{\exp \left(\varepsilon^{2} T\right) \frac{\Phi\left(z_{\alpha}-2 \varepsilon \sqrt{T}\right)}{\alpha}}\right)=g_{2}(\varepsilon),
$$

where $\theta=\left\|\sigma^{-1}(b-r \mathbf{1})\right\|$. By Lemma 3.3,

$$
\lim _{\varepsilon \rightarrow+\infty} f\left(\pi_{\varepsilon}^{*}\right)=\lim _{\varepsilon \rightarrow+\infty} g_{2}(\varepsilon)=+\infty
$$

which completes the proof of assertion (1).

(2) By Proposition 3.1 (3),

$$
\operatorname{EaR}_{2}(\pi, w, T)>0=\operatorname{EaR}_{2}(0, w, T) \text { for all } \pi \neq 0
$$

which implies the conclusion (2).

Proposition 3.4 implies that EaR attains a lower bound of zero for the pure bond strategy. It is bounded from above by $w e^{r T}$ in a risk-neural market and unbounded above otherwise. 


\section{Optimal Portfolio Selection with EaR}

Recall that one model of Markowitz's mean-variance methodology is to minimize the variance of the portfolio return under a given level of the expected portfolio return. Analogously, our dynamic portfolio selection model is to minimize Earnings-at-Risk of the terminal wealth with respect to one of $\rho_{k}$ 's under a given level of the expected terminal wealth. In this paper, we confine the discussion of the case $k=2$. More precisely, we solve the following problem:

$$
(P) \quad \min _{\pi \in \mathbb{R}^{n}} E a R_{2}(\pi) \quad \text { subject to } E\left[W^{\pi}(T)\right] \geq C,
$$

where $C>0$ is a predetermined level of the expected terminal wealth $E\left[W^{\pi}(T)\right]$. We refer the above optimization problem as the mean-EaR problem. Since the pure bond policy yields a deterministic terminal wealth of $w \exp (r T)$, it is natural to assume that the expected wealth level $C$ satisfies the following lower bound condition:

$$
C \geq w \exp (r T)
$$

In fact, if $C<w \exp (r T)$, then, according to Proposition 3.4 (2), the optimal solution of $(P)$ would be the pure bond strategy $\pi=0$.

In the following we derive analytically the best CRP investment strategy; i.e., the optimal solution to portfolio optimization problem $(P)$. As a by-product, we also obtain a closed-form expression for the corresponding mean-EaR efficient frontier.

Theorem 4.1. Assume that $b \neq r \mathbf{1}$. Then the unique optimal policy of problem $(P)$ is

$$
\pi^{*}=\varepsilon^{*} \frac{\left(\sigma \sigma^{\prime}\right)^{-1}(b-r \mathbf{1})}{\left\|\sigma^{-1}(b-r \mathbf{1})\right\|},
$$

where

$$
\varepsilon^{*}=\frac{\ln (C / w)-r T}{\left\|\sigma^{-1}(b-r \mathbf{1})\right\| T} .
$$

The corresponding expected terminal wealth is $E\left[W^{\pi^{*}}(T)\right]=C$ and Earnings-at-Risk is

$$
\operatorname{EaR}_{2}\left(\pi^{*}\right)=C\left[1-\sqrt{\exp \left(\varepsilon^{* 2} T\right) \frac{\Phi\left(z_{\alpha}-2 \varepsilon^{*} \sqrt{T}\right)}{\alpha}}\right] .
$$

Proof. With the help of expression (2.13) for $E a R_{2}$, we rewrite problem $(P)$ as

$$
(P)\left\{\begin{array}{l}
\min \quad w \exp \left(\left(\pi^{\prime}(b-r \mathbf{1})+r\right) T\right)\left[1-\sqrt{\exp \left(\left\|\pi^{\prime} \sigma\right\|^{2} T\right) \frac{\Phi\left(z_{\alpha}-2\left\|\pi^{\prime} \sigma\right\| \sqrt{T}\right)}{\alpha}}\right] \\
\text { s.t. } \quad w \exp \left(\left(\pi^{\prime}(b-r \mathbf{1})+r\right) T\right) \geq C .
\end{array}\right.
$$


If $C=w \exp (r T)$, it is obvious that the pure bond policy $\pi^{*}=0$ is a feasible solution to problem $(P)$, with the global minimal Earnings-at-Risk $E a R_{2}\left(\pi^{*}\right)=0$ by Proposition $3.4(2)$. Hence, $\pi^{*}=0$ is the unique optimal solution of $(P)$, which means that the conclusions asserted are true for this special case. Now we assume that $C>w \exp (r T)$. The feasible set of the problem is

$$
\Pi=\left\{\pi:(b-r \mathbf{1})^{\prime} \pi T \geq \ln \frac{C}{w}-r T\right\} .
$$

Given $\varepsilon>0$, the intersection of $\Pi$ and the ellipsoid $\left\|\pi^{\prime} \sigma\right\|=\varepsilon$ is

$$
\Pi(\varepsilon)=\left\{\pi:\left\|\pi^{\prime} \sigma\right\|=\varepsilon, \quad(b-r \mathbf{1})^{\prime} \pi T \geq \ln \frac{C}{w}-r T\right\} .
$$

The hyperplane $(b-r \mathbf{1})^{\prime} \pi T=\ln \frac{C}{w}-r T$ is tangent to the ellipsoid $\left\|\pi^{\prime} \sigma\right\|=\varepsilon$ if and only if $\varepsilon \theta T=\ln (C / w)-r T$, that is $\varepsilon=\varepsilon^{*}:=\frac{\ln (C / w)-r T}{\theta T}>0$, where $\theta=\left\|\sigma^{-1}(b-r \mathbf{1})\right\|$. Consequently $\Pi(\varepsilon)=\emptyset$ if $\varepsilon<\varepsilon^{*}$ and hence $\Pi=\bigcup_{\varepsilon \geq \varepsilon^{*}} \Pi(\varepsilon)$. Thus problem $(P)$ is equivalent to the following bilevel optimization problem

$$
\left(P^{\prime}\right) \quad \min _{\varepsilon \geq \varepsilon^{*}} \min _{\pi \in \Pi(\varepsilon)} w \exp \left(\left(\pi^{\prime}(b-r \mathbf{1})+r\right) T\right)\left[1-\sqrt{\exp \left(\varepsilon^{2} T\right) \frac{\Phi\left(z_{\alpha}-2 \varepsilon \sqrt{T}\right)}{\alpha}} .\right.
$$

For each fixed $\varepsilon \geq \varepsilon^{*}$, we solve the problem

$$
\min _{\pi \in \Pi(\varepsilon)} w \exp \left(\left(\pi^{\prime}(b-r \mathbf{1})+r\right) T\right)\left[1-\sqrt{\exp \left(\varepsilon^{2} T\right) \frac{\Phi\left(z_{\alpha}-2 \varepsilon \sqrt{T}\right)}{\alpha}}\right]
$$

or equivalently

$$
\min _{\pi \in \Pi(\varepsilon)}(b-r \mathbf{1})^{\prime} \pi T
$$

When $\varepsilon=\varepsilon^{*}$, the optimal solution is the unique tangent point

$$
\pi^{*}=\varepsilon^{*} \frac{\left(\sigma \sigma^{\prime}\right)^{-1}(b-r \mathbf{1})}{\left\|\sigma^{-1}(b-r \mathbf{1})\right\|}
$$

of the hyperplane $(b-r \mathbf{1})^{\prime} \pi T=\ln \frac{C}{w}-r T$ to the ellipsoid $\left\|\pi^{\prime} \sigma\right\|=\varepsilon^{*}$, with $(b-r \mathbf{1})^{\prime} \pi^{*} T=$ $\varepsilon^{*} \theta T$. When $\varepsilon>\varepsilon^{*}, \min \left\{(b-r \mathbf{1})^{\prime} \pi T: \pi \in \Pi(\varepsilon)\right\}=\ln \frac{C}{w}-r T=\varepsilon^{*} \theta T$, and every point on both the hyperplane $(b-r \mathbf{1})^{\prime} \pi T=\ln \frac{C}{w}-r T$ and the ellipsoid $\left\|\pi^{\prime} \sigma\right\|=\varepsilon$ is an optimal solution. Therefore, we obtain the solution of problem $\left(P^{\prime}\right)$ by solving the problem

$$
\min _{\varepsilon \geq \varepsilon^{*}} w \exp \left(\left(\varepsilon^{*} \theta+r\right) T\right)\left[1-\sqrt{\exp \left(\varepsilon^{2} T\right) \frac{\Phi\left(z_{\alpha}-2 \varepsilon \sqrt{T}\right)}{\alpha}}\right] .
$$


Since the function $1-\sqrt{\exp \left(\varepsilon^{2} T\right) \frac{\Phi\left(z_{\alpha}-2 \varepsilon \sqrt{T}\right)}{\alpha}}=1-\sqrt{g_{1}(\varepsilon)}$ is strictly increasing with respect to $\varepsilon$ by Lemma 3.3 , the optimal $\varepsilon$ for the above problem is the unique $\varepsilon^{*}$. This completes the proof.

As an immediate consequence, the analytic result in Theorem 4.1 provides an explicit relation between the optimal Earnings-at-Risk and the expected terminal wealth. Letting $\xi:=E\left[W^{\pi^{*}}(T)\right]$, we have

$$
\operatorname{EaR}_{2}(\xi)=\xi\left[1-\sqrt{\frac{1}{\alpha} \exp \left(\frac{(\ln (\xi / w)-r T)^{2}}{\left\|\sigma^{-1}(b-r \mathbf{1})\right\|^{2} T}\right) \Phi\left(z_{\alpha}-2 \frac{\ln (\xi / w)-r T}{\left\|\sigma^{-1}(b-r \mathbf{1})\right\| \sqrt{T}}\right)}\right]
$$

for $\xi \geq w \exp (r T)$. The above relationship is known as the efficient frontier for the mean-EaR problem in mean-EaR space.

We now make several remarks about the best CRP investment strategy and the mean-EaR efficient frontier derived above.

Remark 4.1. Observe that the best CRP investment strategy is independent of the confidence level $\alpha$. However, the mean-EaR efficient frontier depends on $\alpha$. Smaller $\alpha$ is achieved at the expense of higher risk measured by $E a R_{2}$ in order to maintain the same expected terminal wealth. In other words, for a given level of expected terminal wealth, EaR of the best CRP investment strategy is decreasing in confidence level $\alpha$ when $\alpha<0.5$; see Appendix A for a proof.

Remark 4.2. Expression (4.5) of the efficient frontier also implies that for a given level of expected terminal wealth, EaR of the best CRP investment strategy is strictly decreasing in time horizon $T$, a behavior which is consistent with intuition. The reason for this is that $\operatorname{EaR}_{2}(\xi)$ is compounded by functions $\operatorname{EaR}_{2}(\xi)=\xi\left(1-\sqrt{g_{1}(\varepsilon)}\right)$ and $\varepsilon=$ $(\ln (\xi / w)-r T) /\left(\left\|\sigma^{-1}(b-r \mathbf{1})\right\| T\right)$ and that $g_{1}(\varepsilon)$ is strictly decreasing by Lemma 3.3 and $\varepsilon$ is strictly decreasing in $T$ when $T$ satisfies $\xi \geq \exp (r T)$.

Remark 4.3. The above mean-EaR efficient frontier is obtained by solving the optimization problem $(\mathrm{P})$. Alternatively, the same efficient frontier could have obtained by maximizing the mean terminal wealth for a given level of EaR; i.e.,

$$
\left(P^{\prime}\right) \quad \max _{\pi \in \mathbb{R}^{n}} E\left[W^{\pi}(T)\right] \quad \text { subject to } \operatorname{EaR}_{2}(\pi) \leq C^{\prime},
$$

where $C^{\prime}$ is a given constant.

We now demonstrate a numerical example to end this section. 
Example 4.1. Let the initial wealth be $w=1000$ and assume that the market consists of the bond and just one stock (i.e., $n=1$ ). Assume that the rate of interest of the bond is $r=0.05$, the stock-appreciation rate is $b=0.1$, and the stock-volatility is $\sigma=0.2$. Then $\theta=0.25$. As the level $C$ of the expected terminal wealth we use the terminal wealth of the pure bond policy at $T=5$; that is, $C=1000 \exp (0.05 \times 5)=1284$. Figure 1 shows the dependence of the best CRP investment strategy and the pure stock strategy on the time horizon $T(0<T \leq 5)$. The best CRP investment strategy always contains a short position in bond when $T<2.5$ and a long position in both bond and stock when $T>2.5$. Now we take three different confidence levels: $\alpha=0.01,0.05$ and 0.1 , which imply that the corresponding quantiles are $z_{\alpha}=-2.326,-1.645$ and -1.282 respectively. The EaR of the best CRP investment strategy for different confidence levels and the EaR of the pure stock strategy as functions of the time horizon $T(0<T \leq 5)$ are plotted in Figure 2, where the increasing dash line describes the EaR of the pure stock strategy, and the decreasing dotted line, solid line and bold line describe the EaR of the best CRP investment strategy for confidence levels $\alpha=0.01,0.05$ and 0.1 respectively. The three decreasing lines are even lower than the increasing line after $T$ is large appropriately. Clearly, the higher the confidence level $\alpha$, the lower is the line for EaR of the best CRP investment strategy.

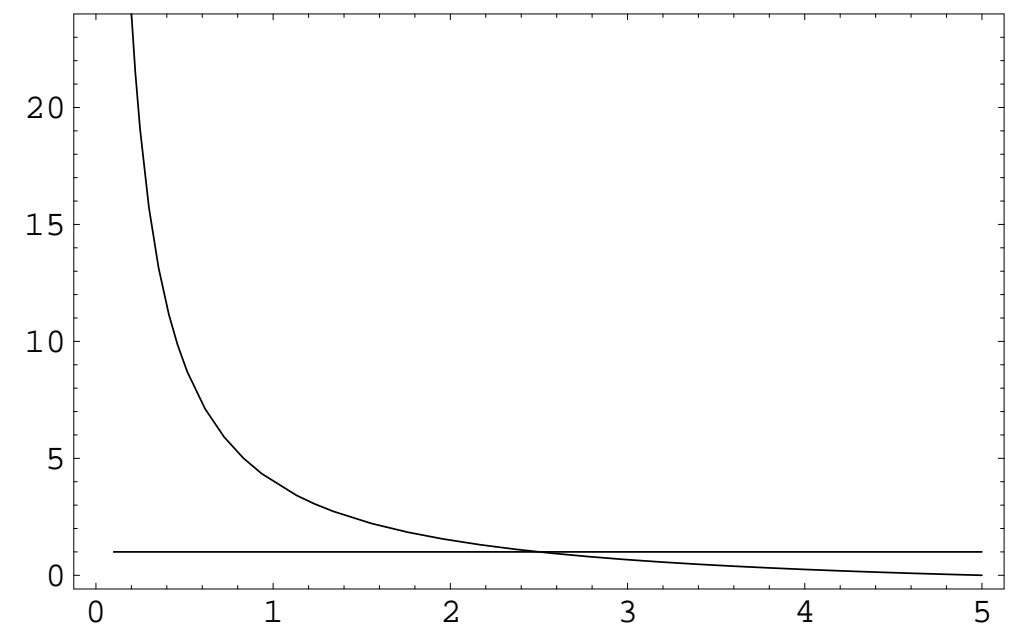

Figure 1: The best CRP and the pure stock strategies as functions of the time horizon $T(0<T \leq 5)$ 


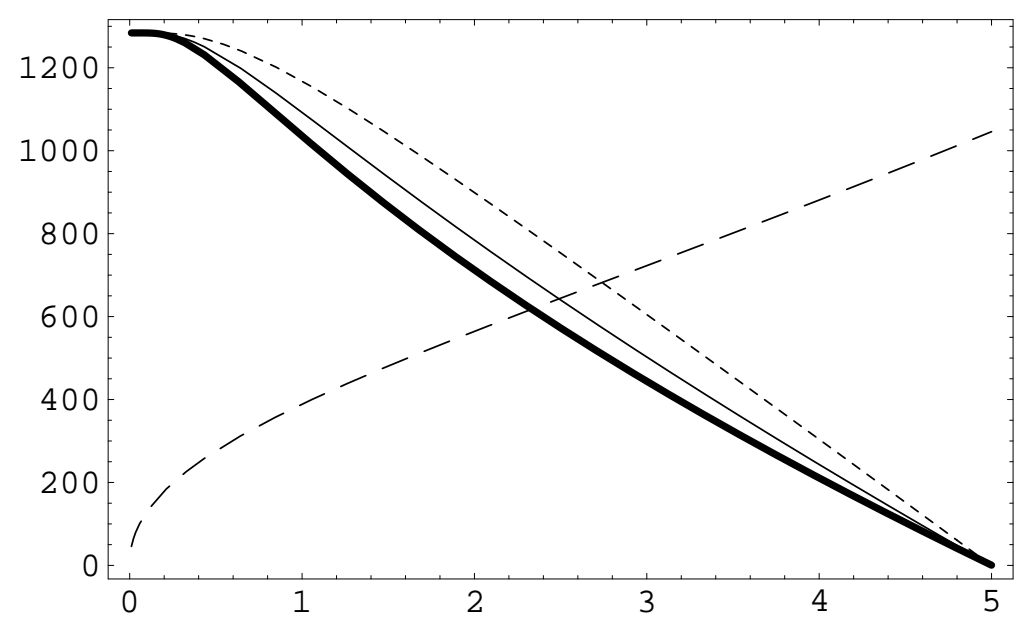

Figure 2: EaR of the best CRP and the pure security strategies as functions of the time horizon $T(0<T \leq 5)$

\section{A Comparison with Mean-Variance, Mean-CaR, and Expected Utility Analyses}

In this section we compare the proposed mean-EaR model to the classical mean-variance portfolio selection model, to the mean-CaR model discussed in Ref. 13, and to the usual expected utility model.

\subsection{A Comparison with Mean-Variance Analysis}

First we focus on a comparison with mean-variance analysis. In particular, we consider the following mean-variance optimization problem:

$$
(\hat{P}) \quad \min _{\pi \in \mathbb{R}^{n}} \operatorname{Var}\left[W^{\pi}(T)\right] \quad \text { subject to } \quad E\left[W^{\pi}(T)\right] \geq C,
$$

where $C$, as in problem $(P)$, is the predetermined level of the expected terminal wealth $E\left[W^{\pi}(T)\right]$ that satisfies condition (4.1).

The solution to the above optimization problem $(\hat{P})$ is summarized in the following theorem. We omit the proof since it is very similar to the proof of Theorem 4.1.

Theorem 5.1. Assume that $b \neq r \mathbf{1}$. Then the unique optimal policy of mean-variance problem $(\hat{P})$ is

$$
\pi^{*}=\varepsilon^{*} \frac{\left(\sigma \sigma^{\prime}\right)^{-1}(b-r \mathbf{1})}{\left\|\sigma^{-1}(b-r \mathbf{1})\right\|}
$$

where

$$
\varepsilon^{*}=\frac{\ln (C / w)-r T}{\left\|\sigma^{-1}(b-r \mathbf{1})\right\| T}
$$


The corresponding expected terminal wealth is $E\left[W^{\pi^{*}}(T)\right]=C$ and variance

$$
\operatorname{Var}\left[W^{\pi^{*}}(T)\right]=C^{2}\left[\exp \left(\varepsilon^{* 2} T\right)-1\right] .
$$

It follows immediately from the above result that the efficient frontier for the meanvariance problem in mean-variance space is given by

$$
\nu=\xi^{2}\left[\exp \left(\frac{[\ln (\xi / w)-r T]^{2}}{\left\|\sigma^{-1}(b-r \mathbf{1})\right\|^{2} T}\right)-1\right] \quad \text { for } \quad \xi \geq w \exp (r T),
$$

where $\nu:=\operatorname{Var}\left[W^{\pi^{*}}(T)\right]$ and $\xi:=E\left[W^{\pi^{*}}(T)\right]$.

It should be pointed out that the continuous-time mean-variance model discussed in Ref. 13 maximizes the expected terminal wealth for a given level of variance of the terminal wealth. Although they also obtained a solution that has the same representation as (5.1), the parameter $\varepsilon^{*}$ however was not obtained explicitly as in (5.2). In fact, in their formulation $\varepsilon^{*}$ is expressed as the unique positive solution to a nonlinear equation. Consequently, they did not obtain the mean-variance efficient frontier explicitly.

An interesting consequence of Theorems 4.1 and 5.1 is that for a given minimum level $C$ of the expected terminal wealth $E\left[W^{\pi}(T)\right]$, the optimal CRP investment strategies for both the mean-EaR and the mean-variance problems are equivalent, as indicated by (4.2) and (5.1). In fact, it can also be shown that similar optimal $\pi^{*}$ can also be obtained if we had considered the risk measure CaR as in the mean-CaR optimization problem. This implies all these risk measures yield similar optimal CRP investment strategies as long as the preselected level $C$ is identical.

The above observation also provides a linkage between the EaR and the variance of terminal wealth. For instance, suppose we fixed the level of EaR. From the mean-EaR efficient frontier (4.5), we derive the highest attainable expected return and hence the optimal portfolio $\pi^{*}$ using (4.2). This in turn allows us to determine the corresponding minimum variance of terminal wealth using (5.3). Similarly, if the level of variance of terminal wealth is given, the mean-variance efficient frontier (5.4) can be used to obtain the corresponding expected terminal wealth and hence the minimum acceptable EaR using (4.4).

We now draw additional insights based on efficient frontiers (4.5) and (5.4) derived respectively from the mean-EaR and mean-variance problems.

Remark 5.1. The global minimal EaR is zero and the minimum EaR portfolio strategy is the pure bond strategy. This is also a consequence of Proposition 3.4. The global 
minimal variance is zero and the minimum variance portfolio strategy is the pure bond strategy.

Remark 5.2. On the efficient frontiers, both EaR and variance are strictly increasing functions of the expected terminal wealth, as to be expected. For the reason, we need only to note that $\operatorname{EaR}_{2}(\xi)$ is a product of two functions. The first function is $\xi$ which is strictly increasing. The second function is $1-\sqrt{g_{1}(\varepsilon)}$ with $\varepsilon=$ $(\ln (\xi / w)-r T) /\left(\left\|\sigma^{-1}(b-r \mathbf{1})\right\| T\right)$. This function is also strictly increasing because $g_{1}(\varepsilon)$ is strictly decreasing by Lemma 3.3 and because $\varepsilon$ is strictly increasing in $\xi$.

Remark 5.3. For the mean-variance efficient frontier, the variance is always a convex function of the expected terminal wealth. For the mean-EaR efficient frontier, the situation is more complicated, depending on the data input. However, at least over a infinite subinterval of the terminal wealth, EaR is a concave function of the expected terminal wealth. See Appendix B for a proof. These facts imply that the marginal risk (measured by variance) of the expected terminal wealth is always increasing on the mean-variance efficient frontier, while the marginal risk (measured by EaR) is decreasing at least on a infinite part of the mean-EaR frontier.

To end this subsection, we consider a numerical example to illustrate the difference between the mean-EaR and the mean-variance efficient frontiers.

Example 5.1. The same as in Example 4.1, we let $n=1, w=1000, T=5, r=0.05, b=$ $0.1, \sigma=0.2$. Then $\theta=0.25$. With these parameters, the mean-variance efficient frontier is plotted in Figure 4 with the mean on the horizontal axis and the variance on the vertical axis. Further, we take three different confidence levels: $\alpha=0.01,0.05$ and 0.1 , which imply that the corresponding quantiles are $z_{\alpha}=-2.326,-1.645$ and -1.282 respectively. Three corresponding mean-EaR efficient frontiers are depicted in Figure 3 with the mean on the horizontal axis and the EaR on the vertical axis. The dash line, the solid line and the bold line describe the mean-EaR efficient frontiers for confidence levels $\alpha=0.01,0.05$ and 0.1 respectively. Clearly, the mean-EaR efficient frontiers are increasing and concave while the mean-variance efficient frontier is increasing and convex. And, the higher the confidence level $\alpha$, the lower is the mean-EaR efficient frontier. 


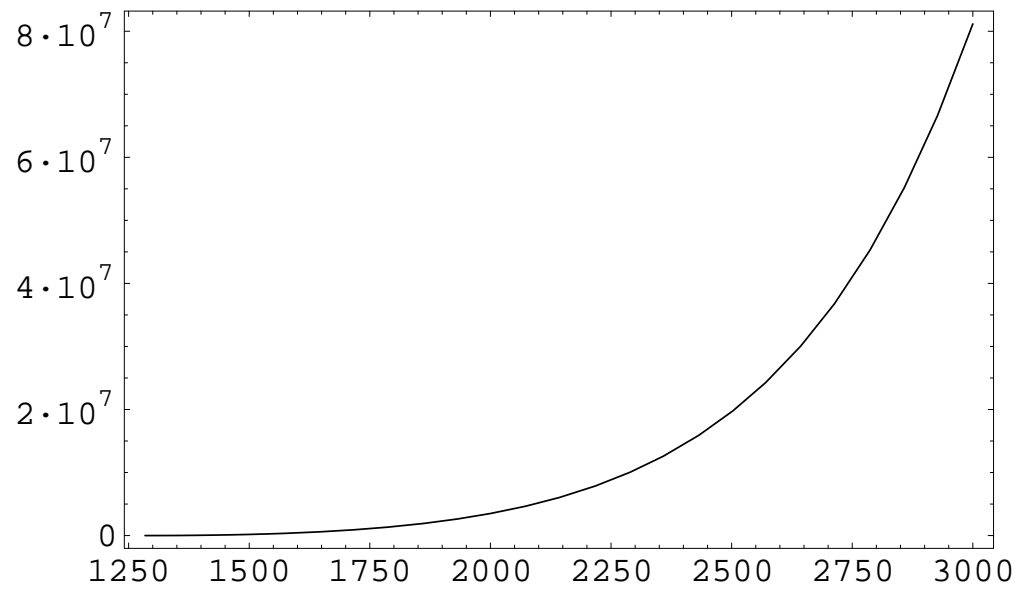

Figure 3: Mean-Variance efficient frontier

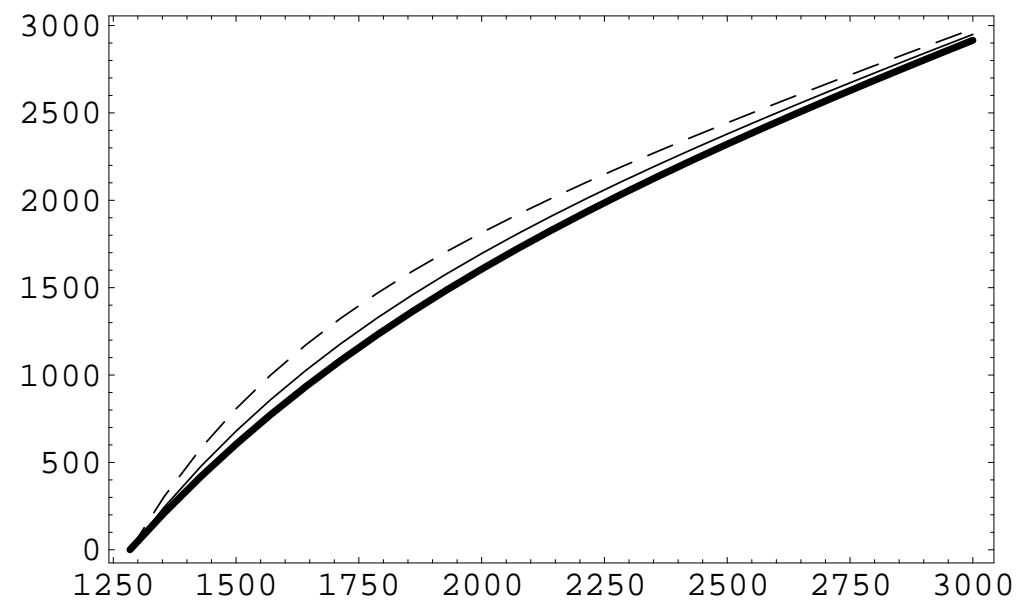

Figure 4: Mean-EaR efficient frontier 


\subsection{A Comparison with Mean-CaR Analysis}

We now turn to a comparison with mean-CaR analysis. The concept of CaR (Capital at Risk) proposed in Ref. 13 is defined as

$$
\begin{aligned}
C a R(\pi) & =w \exp (r T)-\rho_{0}(\pi, w, T) \\
& =w \exp (r T)-w \exp \left(\left(\pi^{\prime}(b-r \mathbf{1})+r-\left\|\pi^{\prime} \sigma\right\|^{2} / 2\right) T+z_{\alpha}\left\|\pi^{\prime} \sigma\right\| \sqrt{T}\right) .
\end{aligned}
$$

They formulated their mean-CaR model as maximizing the expected terminal wealth for a given level of $\mathrm{CaR}$ of the terminal wealth. For the sake of a convenient and easy comparison, as the above we formulate a mean-CaR model by minimizing the $\mathrm{CaR}$ of the terminal wealth for a given level of the expected terminal wealth:

$$
(\check{P}) \quad \min _{\pi \in \mathbb{R}^{n}} C a R(\pi) \quad \text { subject to } \quad E\left[W^{\pi}(T)\right] \geq C,
$$

where $C$, again as in problem $(P)$, is the predetermined level of the expected terminal wealth $E\left[W^{\pi}(T)\right]$ that satisfies condition (4.1).

Using a quite similar derivation as that in the proof of Theorem 4.1, we can also obtain a closed-form solution for problem $(\check{P})$, which is summarized by the following theorem stated without proof.

Theorem 5.2. Assume that $b \neq r \mathbf{1}$. Then the unique optimal policy of mean-CaR problem $(\check{P})$ is

$$
\pi^{*}=\varepsilon^{*} \frac{\left(\sigma \sigma^{\prime}\right)^{-1}(b-r \mathbf{1})}{\left\|\sigma^{-1}(b-r \mathbf{1})\right\|}
$$

where

$$
\varepsilon^{*}=\max \left\{\frac{\ln (C / w)-r T}{\theta T}, \theta+\frac{z_{\alpha}}{\sqrt{T}}\right\}
$$

with $\theta:=\left\|\sigma^{-1}(b-r \mathbf{1})\right\|$.

Based on this result, the efficient frontier for the mean-CaR problem in mean-CaR space is given by

$$
C a R(\xi)=w \exp (r T)-\xi \exp \left(\frac{\ln (\xi / w)-r T}{\theta T}\left(z_{\alpha} \sqrt{T}-\frac{\ln (\xi / w)-r T}{2 \theta}\right)\right)
$$

where

$$
\xi:=E\left[W^{\pi^{*}}(T)\right] \geq \begin{cases}w \exp (r T) & \text { if } \theta \sqrt{T}<\left|z_{\alpha}\right|, \\ w \exp \left(r T+\theta \sqrt{T}\left(\theta \sqrt{T}-\left|z_{\alpha}\right|\right)\right) & \text { otherwise. }\end{cases}
$$


We have seen that a substantial difference of the mean-CaR model from the meanEaR is that, in the case $\theta \sqrt{T} \geq\left|z_{\alpha}\right|$, the best CRP investment strategy is the same, equal to

$$
\pi^{*}=\left(\theta+\frac{z_{\alpha}}{\sqrt{T}}\right) \frac{\left(\sigma \sigma^{\prime}\right)^{-1}(b-r \mathbf{1})}{\left\|\sigma^{-1}(b-r \mathbf{1})\right\|}
$$

for all $C$ that satisfies

$$
w \exp (r T) \leq C \leq w \exp \left(r T+\theta \sqrt{T}\left(\theta \sqrt{T}-\left|z_{\alpha}\right|\right)\right)
$$

Corresponding to these $C$, the part of the efficient frontier for the mean-CaR problem degenerates to only one point in mean-CaR space. The whole efficient frontier starts only from this point where $\xi=w \exp \left(r T+\theta \sqrt{T}\left(\theta \sqrt{T}-\left|z_{\alpha}\right|\right)\right)$.

We have also noted that a common fact for the mean-variance, the mean-CaR, and the mean-CaR models is that their efficient frontiers only depend on the stocks via the norm $\left\|\sigma^{-1}(b-r \mathbf{1})\right\|$. There is no explicit dependence on the number of different stocks. Therefore Theorems 4.1, 5.1 and 5.2 can be interpreted as a kind of mutual fund theorems since there is no difference between investment in our multi-stock market and a market consisting of the bond and just one stock with appropriate market coefficients $b$ and $\sigma$. This was observed by Emmer, Klüppelberg and Korn in Ref. 13 for their mean-CaR model.

\subsection{A Comparison with Expected Utility Analysis}

How does an investor's optimal CRP investment strategy change when he or she, previously using mean-EaR criterion, decides to use expected utility criterion?

We now show that, for risk-averse investors with constant relative risk-aversion, the model of maximizing expected utility of terminal wealth results in the same optimal CRP investment strategy as the mean-EaR model with appropriate levels of expected terminal wealth.

Suppose that an investor has a strictly increasing utility function $u: \mathbb{R}_{+} \rightarrow \mathbb{R}$ displaying constant relative risk-aversion and maximizes expected utility of terminal wealth. The investor's portfolio selection problem is

$$
(U P) \quad \max _{\pi \in \mathbb{R}^{n}} E\left[u\left(W^{\pi}(T)\right)\right] .
$$

Let $u(W)=W^{\gamma} / \gamma, \gamma<1$ and $\gamma \neq 0$ or $u(W)=\ln (W)$ (the limiting case when $\gamma$ approaches 0) where $-u^{\prime \prime}(W) W / u^{\prime}(W)=1-\gamma$ is Arrow-Pratt measure of relative risk 
aversion. Then, in this particular case,

$$
E\left[u\left(W^{\pi}(T)\right)\right]=w^{\gamma} \exp \left(\gamma\left(\pi^{\prime}(b-r \mathbf{1})+r-(1-\gamma)\left\|\pi^{\prime} \sigma\right\|^{2} / 2\right) T\right) / \gamma
$$

Theorem 5.3. Assume that $b \neq r \mathbf{1}$. Then the unique optimal policy of expected utility maximization problem $(U P)$ is

$$
\pi^{*}=\left(\sigma \sigma^{\prime}\right)^{-1}(b-r \mathbf{1}) /(1-\gamma)
$$

Proof. The expected utility maximization problem is equivalent to

$$
\max _{\pi \in \mathbb{R}^{n}} \pi^{\prime}(b-r \mathbf{1})-(1-\gamma)\left\|\pi^{\prime} \sigma\right\|^{2} / 2,
$$

which can further be written as

$$
\max _{\varepsilon \geq 0} \max _{\left\|\pi^{\prime} \sigma\right\|=\varepsilon} \pi^{\prime}(b-r \mathbf{1})-(1-\gamma) \varepsilon^{2} / 2 .
$$

The optimal solution of the inner level optimization problem is given by Proposition 3.4 and is

$$
\pi_{\varepsilon}^{*}=\varepsilon \frac{\left(\sigma \sigma^{\prime}\right)^{-1}(b-r \mathbf{1})}{\left\|\sigma^{-1}(b-r \mathbf{1})\right\|}
$$

with

$$
\pi_{\varepsilon}^{* \prime}(b-r \mathbf{1})=\varepsilon \theta .
$$

Therefore, what is left is to solve the problem

$$
\max _{\varepsilon \geq 0} \varepsilon \theta-(1-\gamma) \varepsilon^{2} / 2
$$

Clearly, when $\gamma<1$ its optimal solution is

$$
\varepsilon^{*}=\theta /(1-\gamma)
$$

Thus, the optimal CRP strategy of expected utility maximization problem $(U P)$ is given by (5.8).

Thus, the optimal CRP strategy of expected utility maximization problem $(U P)$ is the same as the one of mean-EaR problem $(P)$ when $C=w \exp \left(r T+\theta^{2} T /(1-\gamma)\right)$.

\section{Conclusions}

In this paper, we have introduced a risk concept known as Earnings-at-Risk to replace the variance in mean-variance analysis, derived closed-form solutions to a mean-EaR 
dynamic portfolio optimization problem under the Black-Scholes setting, and compared our mean-EaR analysis to the classical mean-variance analysis, to the mean-CaR analysis, and to the expected utility analysis. Our closed-form explicit formulae of optimal CRP investment strategies to the mean-EaR, the mean-CaR, and the mean-variance models facilitates the calculation and allow us to exactly and explicitly describe the efficient frontiers for these models and to analyze economic implications. Our models having the same constraint make it easier not only to derive closed-form solutions but also to compare solutions to different portfolio optimization problems. Moreover, our solution method and the idea of this paper also provide useful insights for some other dynamic portfolio optimization problems such as the mean-VaR, Safety-First proposed by Roy in Ref. 17, and other kinds of mean-EaR as well as mean-CaR type problems.

\section{Appendix A}

For any given level of expected terminal wealth, EaR of the best CRP investment strategy is decreasing in confidence level $\alpha$ when $\alpha<0.5$.

According to (4.4), it suffices to show that the function $\Phi\left(z_{\alpha}-t\right) / \alpha$ of $\alpha$ is increasing when $\alpha<0.5$ for any given $t \geq 0$. To this end, we consider the function $\psi(t):=$ $\varphi\left(z_{\alpha}-t\right) / \Phi\left(z_{\alpha}-t\right)$ for $t \geq 0$. We have

$$
\begin{aligned}
\psi^{\prime}(t) & =\frac{-\varphi^{\prime}\left(z_{\alpha}-t\right) \Phi\left(z_{\alpha}-t\right)+\varphi^{2}\left(z_{\alpha}-t\right)}{\Phi^{2}\left(z_{\alpha}-t\right)} \\
& =\frac{\left(z_{\alpha}-t\right) \varphi\left(z_{\alpha}-t\right) \Phi\left(z_{\alpha}-t\right)+\varphi^{2}\left(z_{\alpha}-t\right)}{\Phi^{2}\left(z_{\alpha}-t\right)} \\
& =\frac{\varphi\left(z_{\alpha}-t\right)\left[\varphi\left(t-z_{\alpha}\right)-\left(t-z_{\alpha}\right) \Phi\left(z_{\alpha}-t\right)\right]}{\Phi^{2}\left(z_{\alpha}-t\right)} \\
& >0
\end{aligned}
$$

by Lemma 3.2 with $x=t-z_{\alpha}>0$. Hence, $\psi(t)$ is strictly increasing when $t \geq 0$. This implies that

$$
\frac{\varphi\left(z_{\alpha}-t\right)}{\Phi\left(z_{\alpha}-t\right)}>\frac{\varphi\left(z_{\alpha}\right)}{\Phi\left(z_{\alpha}\right)}=\frac{\varphi\left(z_{\alpha}\right)}{\alpha} \quad \text { for } \quad t>0
$$

Since $\Phi\left(z_{\alpha}\right)=\alpha$, we have $\varphi\left(z_{\alpha}\right) z_{\alpha}^{\prime}=1$; i.e., $z_{\alpha}^{\prime}=1 / \varphi\left(z_{\alpha}\right)$. This together with the above expression implies that

$$
\begin{aligned}
\left(\frac{\Phi\left(z_{\alpha}-t\right)}{\alpha}\right)^{\prime} & =\frac{\alpha \varphi\left(z_{\alpha}-t\right) z_{\alpha}^{\prime}-\Phi\left(z_{\alpha}-t\right)}{\alpha^{2}} \\
& =\frac{1}{\alpha^{2}}\left[\frac{\alpha \varphi\left(z_{\alpha}-t\right)}{\varphi\left(z_{\alpha}\right)}-\Phi\left(z_{\alpha}-t\right)\right] \geq 0
\end{aligned}
$$


for any $t \geq 0$. It follows that the function $\Phi\left(z_{\alpha}-t\right) / \alpha$ of $\alpha$ is increasing when $\alpha<0.5$ for any given $t \geq 0$.

\section{Appendix B}

The mean-variance efficient frontier $\nu(\xi)$ given by (5.4) is convex on the whole interval $[w \exp (r T),+\infty)$ while the mean-EaR efficient frontier $\operatorname{EaR}_{2}(\xi)$ expressed by (4.5) is concave at least on a infinite subinterval of the expected terminal wealth $\xi$.

For simplicity, we denote

$$
\begin{aligned}
e^{*} & =\exp \left(\frac{(\ln (\xi / w)-r T)^{2}}{\left\|\sigma^{-1}(b-r \mathbf{1})\right\|^{2} T}\right), \\
\Phi & =\Phi\left(z_{\alpha}-2 \frac{\ln (\xi / w)-r T}{\left\|\sigma^{-1}(b-r \mathbf{1})\right\| \sqrt{T}}\right), \\
\varphi & =\varphi\left(z_{\alpha}-2 \frac{\ln (\xi / w)-r T}{\left\|\sigma^{-1}(b-r \mathbf{1})\right\| \sqrt{T}}\right), \\
\theta & =\left\|\sigma^{-1}(b-r \mathbf{1})\right\| .
\end{aligned}
$$

Using the formula $(f g)^{\prime \prime}=f^{\prime \prime} g+2 f^{\prime} g^{\prime}+f g^{\prime \prime}$, expression (5.4) leads to

$$
\begin{aligned}
& \frac{d^{2} \nu}{d \xi^{2}}=2\left(e^{\cdot}-1\right)+4 \xi e^{\cdot} \frac{2\left(\ln \frac{\xi}{w}-r T\right)}{\theta^{2} T \xi}+ \\
& \xi^{2}\left[e^{\cdot}\left(\frac{2\left(\ln \frac{\xi}{w}-r T\right)}{\theta^{2} T \xi}\right)^{2}+2 e^{\cdot} \frac{1-\left(\ln \frac{\xi}{w}-r T\right)}{\theta^{2} T \xi^{2}}\right] \\
& =2\left(e^{\cdot}-1\right)+6 e^{\cdot} \frac{\ln \frac{\xi}{w}-r T}{\theta^{2} T \xi}+4 e^{\cdot}\left(\frac{\ln \frac{\xi}{w}-r T}{\theta^{2} T}\right)^{2}+2 e^{\cdot} \frac{1}{\theta^{2} T} \\
& >0
\end{aligned}
$$

for $\xi \geq w \exp (r T)$. This implies that $\nu(\xi)$ is convex on $[w \exp (r T),+\infty)$. For expression (4.5), since

$$
\begin{aligned}
& \left(1-\sqrt{\frac{e^{\bullet} \Phi}{\alpha}}\right)^{\prime} \\
= & -\frac{1}{2 \sqrt{e^{\bullet} \Phi / \alpha} \alpha}\left[e^{\cdot} \Phi \frac{2\left(\ln \frac{\xi}{w}-r T\right)}{\theta^{2} T \xi}+e^{*} \varphi \cdot\left(-\frac{2}{\theta \sqrt{T} \xi}\right)\right] \\
= & -\sqrt{\frac{e^{*}}{\alpha \Phi}} \frac{1}{\xi}\left[\Phi \frac{\ln \frac{\xi}{w}-r T}{\theta^{2} T}-\varphi \frac{1}{\theta \sqrt{T}}\right]
\end{aligned}
$$


and

$$
\begin{aligned}
& \left(1-\sqrt{\frac{e^{\cdot} \Phi}{\alpha}}\right)^{\prime \prime} \\
& =-\left(2 \sqrt{\frac{e^{*}}{\alpha \Phi}}\right)^{-1} \frac{e^{\cdot \frac{2\left(\ln \frac{\xi}{w}-r T\right)}{\theta^{2} T \xi} \Phi-e^{*} \varphi \cdot\left(-\frac{2}{\theta \sqrt{T} \xi}\right)}}{\alpha \Phi^{2}} \frac{1}{\xi}\left[\Phi \frac{\ln \frac{\xi}{w}-r T}{\theta^{2} T}-\varphi \frac{1}{\theta \sqrt{T}}\right] \\
& -\sqrt{\frac{e^{*}}{\alpha \Phi}}\left(-\frac{1}{\xi^{2}}\right)\left[\Phi \frac{\ln \frac{\xi}{w}-r T}{\theta^{2} T}-\varphi \frac{1}{\theta \sqrt{T}}\right] \\
& -\sqrt{\frac{e^{*}}{\alpha \Phi}} \frac{1}{\xi}\left[\varphi \frac{\ln \frac{\xi}{w}-r T}{\theta^{2} T} \frac{-2}{\theta \sqrt{T} \xi}+\Phi \frac{1}{\theta^{2} T \xi}+\frac{-2}{\theta \sqrt{T} \xi}\left(z_{\alpha}-2 \frac{\ln (\xi / w)-r T}{\theta \sqrt{T}}\right) \varphi \frac{1}{\theta \sqrt{T}}\right] \\
& =-\sqrt{\frac{e^{*}}{\alpha \Phi}} \frac{1}{\xi^{2}} \frac{\left(\ln \frac{\xi}{w}-r T\right)^{2} \Phi^{2}-\theta^{2} T \varphi^{2}}{\left(\theta^{2} T\right)^{2} \Phi}+\sqrt{\frac{e^{*}}{\alpha \Phi}} \frac{1}{\xi^{2}}\left[\Phi \frac{\ln \frac{\xi}{w}-r T}{\theta^{2} T}-\varphi \frac{1}{\theta \sqrt{T}}\right] \\
& -\sqrt{\frac{e^{*}}{\alpha \Phi}} \frac{1}{\xi^{2}}\left[\frac{2\left(\ln \frac{\xi}{w}-r T\right) \varphi}{\theta^{2} T \cdot \theta \sqrt{T}}+\frac{\Phi-2 z_{\alpha} \varphi}{\theta^{2} T}\right] \text {, }
\end{aligned}
$$

we have

$$
\begin{aligned}
\operatorname{EaR}_{2}^{\prime \prime}(\xi)= & {\left[\xi\left(1-\sqrt{\frac{e^{\bullet} \Phi}{\alpha}}\right)\right]^{\prime \prime}=2\left(1-\sqrt{\frac{e^{\bullet} \Phi}{\alpha}}\right)^{\prime}+\xi\left(1-\sqrt{\frac{e^{\bullet} \Phi}{\alpha}}\right)^{\prime \prime} } \\
= & -\sqrt{\frac{e^{\bullet}}{\alpha \Phi}} \frac{1}{\theta^{2} T \xi}\left[\frac{\Phi}{\theta^{2} T}\left(\ln \frac{\xi}{w}-r T\right)^{2}+\frac{2 \varphi+\theta \sqrt{T} \Phi}{\theta \sqrt{T}}\left(\ln \frac{\xi}{w}-r T\right)+\right. \\
& \left.\Phi-\left(\theta \sqrt{T}+\frac{\varphi}{\Phi}+2 z_{\alpha}\right) \varphi\right] .
\end{aligned}
$$

We consider the term, [...], in the big bracket of the above expression. Let $t:=$ $\frac{\ln (\xi / w)-r T}{\theta \sqrt{T}}$. Then it can be rewritten as

$$
\begin{aligned}
& {[\ldots] } \\
= & \Phi\left(z_{\alpha}-2 t\right) t^{2}+\left[2 \varphi\left(z_{\alpha}-2 t\right)+\theta \sqrt{T} \Phi\left(z_{\alpha}-2 t\right)\right] t+ \\
& \Phi\left(z_{\alpha}-2 t\right)-\frac{\varphi^{2}\left(z_{\alpha}-2 t\right)}{\Phi\left(z_{\alpha}-2 t\right)}-\left(\theta \sqrt{T}+2 z_{\alpha}\right) \varphi\left(z_{\alpha}-2 t\right) \\
= & \Phi\left(z_{\alpha}-2 t\right)\left(t^{2}+\theta \sqrt{T} t+1\right)+\varphi\left(z_{\alpha}-2 t\right)\left(2 t-\theta \sqrt{T}-2 z_{\alpha}\right)-\frac{\varphi^{2}\left(z_{\alpha}-2 t\right)}{\Phi\left(z_{\alpha}-2 t\right)} .
\end{aligned}
$$


Using the first inequality of Lemma 3.2, it follows that

$$
\begin{aligned}
& {[\ldots] } \\
> & {\left[\frac{1}{2 t-z_{\alpha}}-\frac{1}{\left(2 t-z_{\alpha}\right)^{3}}\right] \varphi\left(z_{\alpha}-2 t\right)\left(t^{2}+\theta \sqrt{T} t+1\right)+} \\
& \varphi\left(z_{\alpha}-2 t\right)\left(2 t-\theta \sqrt{T}-2 z_{\alpha}\right)-\frac{\left(2 t-z_{\alpha}\right)^{3}}{\left(2 t-z_{\alpha}\right)^{2}-1} \varphi\left(z_{\alpha}-2 t\right) \\
= & \frac{\varphi\left(z_{\alpha}-2 t\right)}{\left(2 t-z_{\alpha}\right)^{3}\left[\left(2 t-z_{\alpha}\right)^{2}-1\right]}\left\{\left[\left(2 t-z_{\alpha}\right)^{2}-1\right]^{2}\left(t^{2}+\theta \sqrt{T} t+1\right)-\right. \\
& \left.\left(2 t-z_{\alpha}\right)^{6}+\left(2 t-z_{\alpha}\right)^{3}\left[\left(2 t-z_{\alpha}\right)^{2}-1\right]\left(2 t-\theta \sqrt{T}-2 z_{\alpha}\right)\right\} .
\end{aligned}
$$

In the bracket $\{\ldots\}$, the highest power term is $t^{6}$ and its coefficient is 16 . Therefore, when $\xi$ and hence when $t$ is large sufficiently, [...] $>0$ and hence $\operatorname{EaR}_{2}^{\prime \prime}(\xi)<0$. This means that $\operatorname{EaR}_{2}(\xi)$ is concave on a infinite subinterval of $\xi$.

\section{References}

1. Markowitz, H., Portfolio Selection, The Journal of Finance, Vol. 7, pp. 77-91, 1952.

2. Merton, R. C., Lifetime Portfolio Selection under Uncertainty: The ContinuousTime Case, Review of Economics and Statistics, Vol. 51, pp. 247-256, 1969.

3. Merton, R. C., Optimum Consumption and Portfolio Rules in a ContinuousTime Model, Journal of Economic Theory, Vol. 3, pp. 373-413, 1971.

4. Samuelson, P. A., Lifetime Portfolio Selection by Dynamic Stochastic Propramming, The Review of Economics and Statistics, Vol. 51, pp. 239-246, 1969.

5. Smith, K., A Transition Model for Portfolio Revision, Journal of Finance, Vol. 22, pp. 425-439, 1967.

6. Chen, A., Jen, C., and Zionts, S., The Optimal Portfilio Revision Policy, Journal of Business, Vol. 44, pp. 51-61, 1971.

7. Li D., and NG, W. L., Optimal Dynamic Portfolio Selection: Multiperiod MeanVariance Formulation, Mathematical Fiance, Vol. 10, pp. 387-406, 2000.

8. Zhou, X. Y., and Li, D., Continuous-Time Mean-Variance Portfolio Selection: A Stochastic Lq Framework, Applied Mathematics and Optimization, Vol. 42, pp. 19-33, 2000.

9. Jorion, P., Value at Risk: The New Benchmark for Controlling Market Risk, McGraw-Hill, New York, 1997. 
10. Artzner, P., Delbaen, F., Eber, J., and Heath, D., Coherence measures of risk, Mathematical Finance, Vol. 9, pp. 203-228, 1999.

11. Basak, S., and Şhapiro, A., Value-at-Risk-Based Risk Management: Optimal Policies and Asset Prices, The Review of Financial Studies, Vol. 14, pp. 371-405, 2001.

12. Emmer, S., Klüppelberg, C., and Korn, R., Optimal Portfolios with Bounded Downside Risks, Working Paper (2000), http://www-m4.mathematik.tu-muenchen. $\mathrm{de} / \mathrm{m} 4 /$ pers/cklu/cklu.shtml

13. Emmer, S., Klüppelberg, C., and Korn, R., Optimal Portfolios with Bounded Capital-at-Risk, Mathematical Finance, Vol. 11, pp. 365-384, 2001.

14. Cover, T. M., Universal Portfolios, Mathematical Finance, Vol. 1, pp. 1-29, 1991.

15. Helmbold, D. P., Schapire, R. E., Singer, Y., and Warmuth, M. K., On-Line Portfolio Selection Using Multiplicative Updates, Mathematical Finance, Vol. 8, pp. 325-347, 1998.

16. Gänssler, P., and Stute, W., Wahrscheinlichkeitstheorie, Springer, Berlin, 1977.

17. Roy, A. D., Satety-First and the Holding of Assets, Econometrica, Vol. 20, pp. 431-449, 1952. 